Proceeding Paper

\title{
Biocompatibility and Cellular Uptake of Fluorescent Chitosan Nanohydrogels in Murine Macrophages and B Lymphocytes ${ }^{+}$
}

\author{
Sorina Nicoleta Voicu ${ }^{1,}$, Miruna-Silvia Stan ${ }^{1, *}$, Ionela Cristina Nica ${ }^{1}$, Mihaela Balas ${ }^{1}$, Juliette Moreau ${ }^{2}$, \\ Cyril Cadiou ${ }^{2}$, Maïté Callewaert ${ }^{2}$, Françoise Chuburu ${ }^{2}$ and Anca Dinischiotu ${ }^{1}$
}

Citation: Voicu, S.N.; Stan, M.-S.; Nica, I.C.; Balas, M.; Moreau, J.; Cadiou, C.; Callewaert, M.; Chuburu, F.; Dinischiotu, A. Biocompatibility and Cellular Uptake of Fluorescent Chitosan Nanohydrogels in Murine Macrophages and B Lymphocytes. Mater. Proc. 2021, 4, 10. https:// doi.org/10.3390/IOCN202007851

Academic Editors: Ana María Díez-Pascual, Antonio Di Bartolomeo and Guanying Chen

Published: 11 November 2020

Publisher's Note: MDPI stays neutral with regard to jurisdictional claims in published maps and institutional affiliations.

Copyright: $(2020$ by the authors. Licensee MDPI, Basel, Switzerland. This article is an open access article distributed under the terms and conditions of the Creative Commons Attribution (CC BY) license (http://creativecommons.org/licenses/by/4.0/).
1 Department of Biochemistry and Molecular Biology, Faculty of Biology, University of Bucharest, 91-95 Splaiul Independentei, 050095 Bucharest, Romania; cristinai.nica@gmail.com (I.C.N.); mihaela.balas@bio.unibuc.ro (M.B.); ancadinischiotu@yahoo.com (A.D.)

2 Institute of Molecular Chemistry of Reims, UMR CNRS 7312, University of Reims Champagne Ardenne, 51100 Reims, France; juliette.moreau@univ-reims.fr (J.M.); cyril.cadiou@univ-reims.fr (C.C.); maite.callewaert@univ-reims.fr (M.C.); francoise.chuburu@univ-reims.fr (F.C.)

* Correspondence: sorina.voicu@bio.unibuc.ro (S.N.V.); miruna.stan@bio.unibuc.ro (M.-S.S.)

+ Presented at the 2nd International Online-Conference on Nanomaterials, 15-30 November 2020; Available online: https://iocn2020.sciforum.net/.

\begin{abstract}
Due to their intrinsic viscosity and hydrophilicity, nanohydrogel systems are used to significantly increase the efficiency of commercial contrast agents for MRI and thus effectively improve the sensitivity of the MRI technique. Since chitosan (CS) is a biocompatible polysaccharide frequently used in biomedical applications, we aimed to prepare chitosan nanohydrogels (NGs) by ionic gelation, the polysaccharide being further grafted with rhodamine (RBITC) and fluorescein isothiocyanate (FITC). In this way, the cytotoxic effect of different concentrations $(5,15,30,60$, and $120 \mu \mathrm{g} / \mathrm{mL}$ ) of the fluorescent CS-FITC and CS-RBITC NGs was investigated by assessing the plasma membrane integrity and the metabolic activity of RAW 264.7 murine macrophages and A20 mouse lymphoma B cells following exposure for 6 and $24 \mathrm{~h}$. The cell viability (MTT assay) and lactate dehydrogenase activity were analyzed by spectrophotometric methods, while cellular uptake was observed by fluorescence microscopy. Our results showed that the exposure to CS-FITC and CS-RBITC NGs for 6 and $24 \mathrm{~h}$ did not induce significant changes to RAW 264.7 and A20 cells compared to control, proving a good nanogel biocompatibility for both cell lines. In addition, the fluorescence microscopy showed that cellular uptake was quite rapid and efficient for the NGs tested. Taking all of these into consideration, we can conclude that all types of nanohydrogels were biocompatible, being internalized in both cell types with predominantly cytoplasmic localization.
\end{abstract}

Keywords: nanohydrogels; cellular uptake; murine macrophages; chitosan; B lymphocytes

\section{Introduction}

Due to their intrinsic viscosity and hydrophilicity, nanohydrogel systems are used to significantly increase the efficiency of commercial contrast agents for MRI and thus effectively improve the sensitivity of the MRI technique. Since chitosan (CS) is a biocompatible polysaccharide frequently used in biomedical applications, we aimed to prepare chitosan nanohydrogels (NGs) by ionic gelation, the polysaccharide being further grafted with rhodamine (RBITC) and fluorescein isothiocyanate (FITC).

\section{Materials and Methods}

The cytotoxic effect of different concentrations $(5,15,30,60$, and $120 \mu \mathrm{g} / \mathrm{mL})$ of the fluorescent CS-FITC and CS-RBITC NGs was investigated by assessing the plasma membrane integrity and the metabolic activity of RAW 264.7 murine macrophages and A20 mouse lymphoma B cells following exposure for 6 and $24 \mathrm{~h}$. 


\section{Results}

The cell viability (MTT assay) and lactate dehydrogenase activity were analyzed by spectrophotometric methods, while cellular uptake was observed by fluorescence microscopy. Our results showed that the exposure to CS-FITC and CS-RBITC NGs for 6 and $24 \mathrm{~h}$ did not induce significant changes to RAW 264.7 and A20 cells compared to control, proving a good nanogel biocompatibility for both cell lines. In addition, the fluorescence microscopy showed that cellular uptake was quite rapid and efficient for the NGs tested (Figure 1).

\section{RAW 264.7 cells}

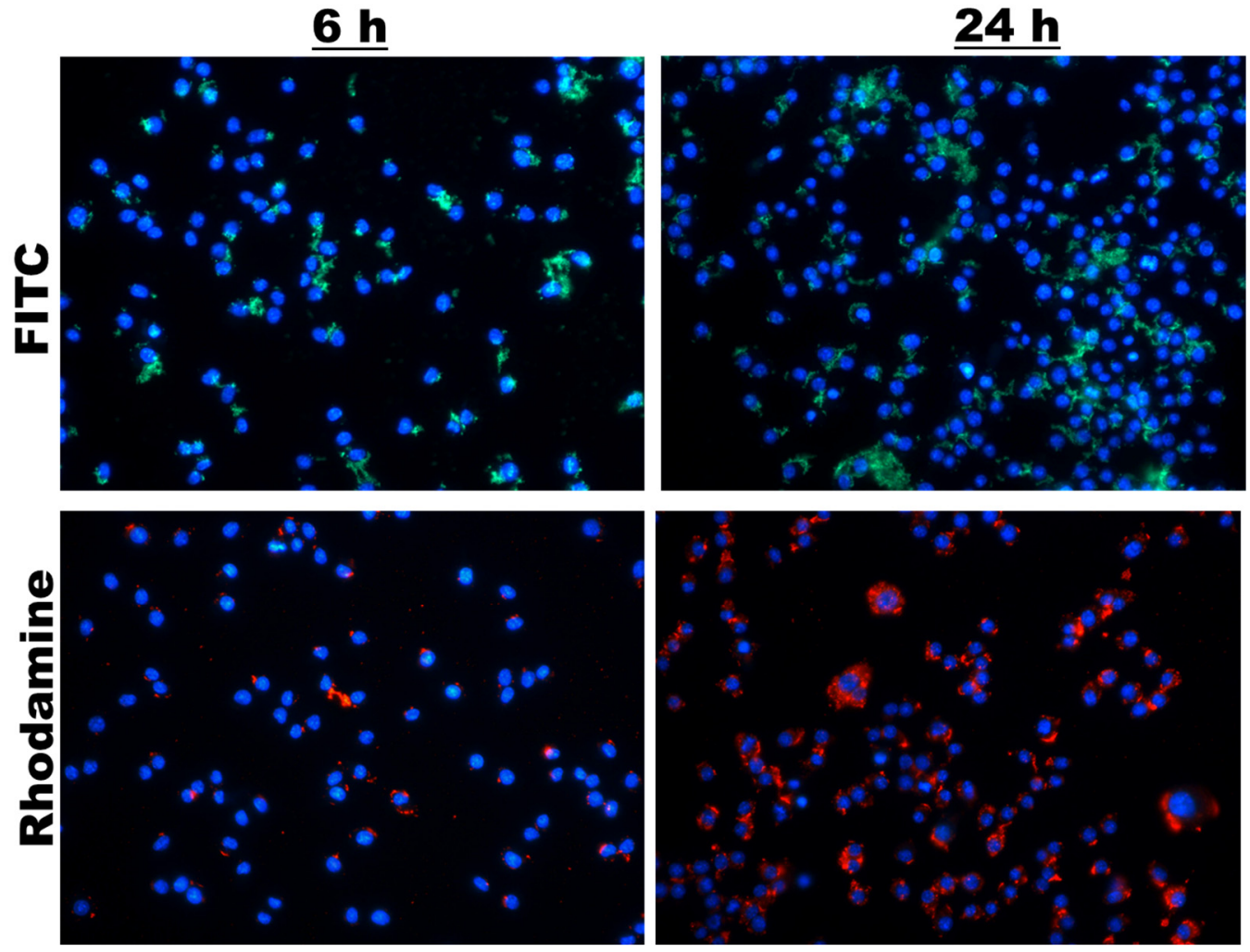



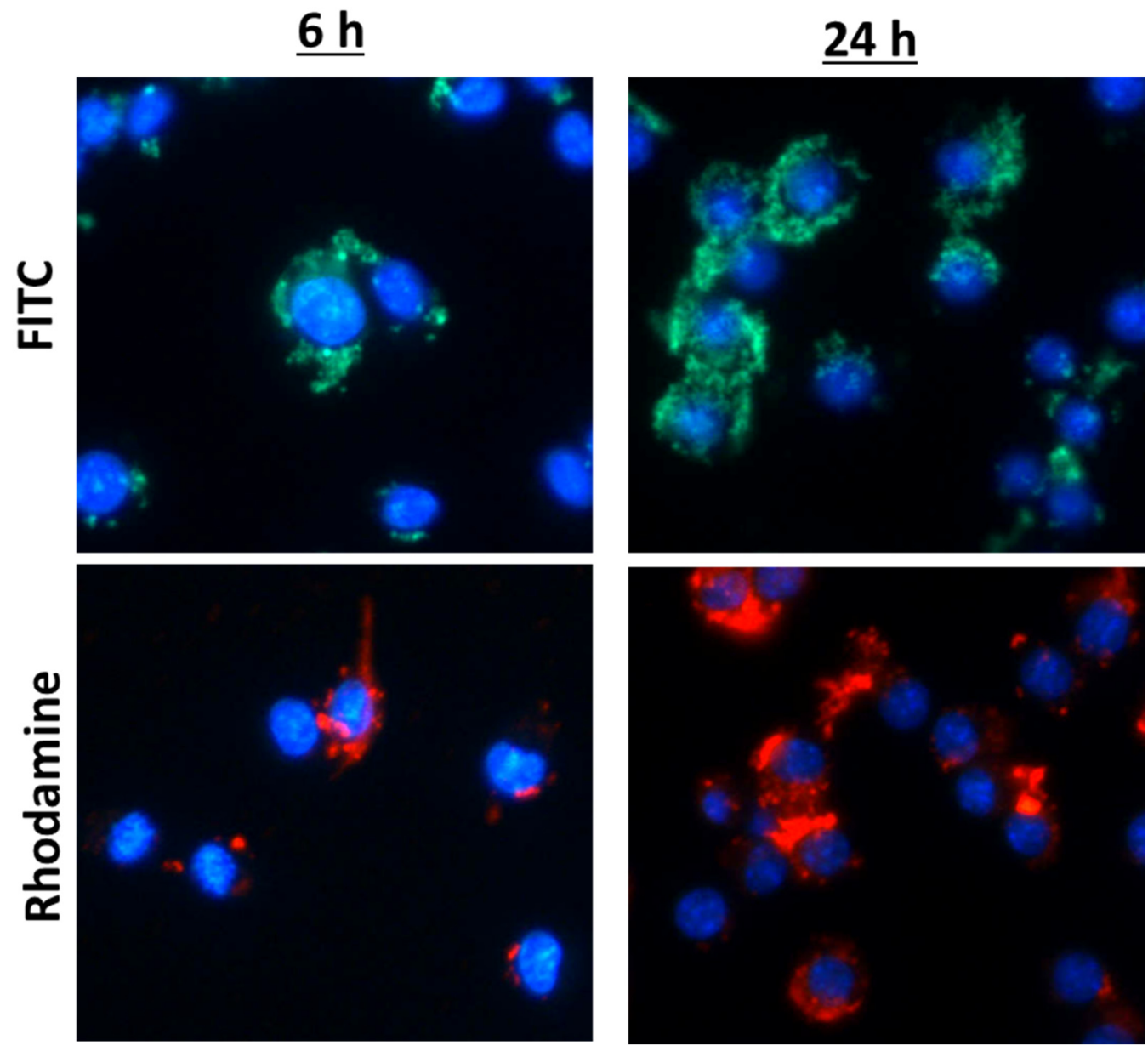

Figure 1. Fluorescence images showing the nuclei of RAW 264.7 cells stained in blue with DAPI solution and the nanogels having rhodamine or fluorescein isothiocyanate (FITC) molecules grafted on chitosan backbone.

\section{Conclusions}

Taking all of these into consideration, we can conclude that all types of nanohydrogels were biocompatible, being internalized in both cell types with predominantly cytoplasmic localization.

Funding: This work was supported by a grant of the Romanian Ministry of Research and Innovation, CCCDI-UEFISCDI, project number PN-III-P3-3.1-PM-RO-FR-2019-0204/6 BM/2019, within PNCDI III.

Conflicts of Interest: The authors declare no conflict of interest. The funders had no role in the design of the study; in the collection, analyses, or interpretation of data; in the writing of the manuscript, or in the decision to publish the results. 